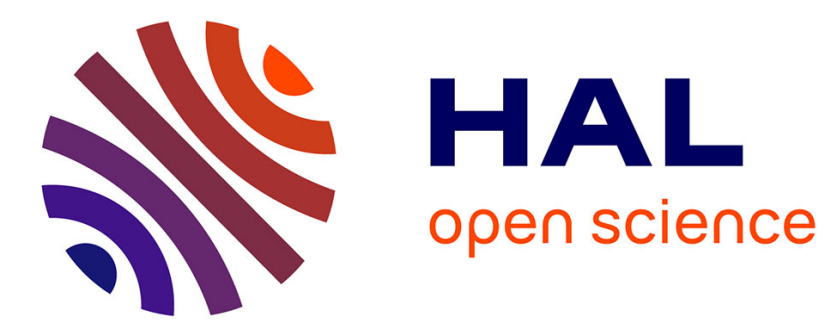

\title{
A New Test for Chaos and Determinism based on Symbolic Dynamics
}

Mariano Matilla-García, Manuel Ruiz Marín

\section{To cite this version:}

Mariano Matilla-García, Manuel Ruiz Marín. A New Test for Chaos and Determinism based on Symbolic Dynamics. Journal of Economic Behavior and Organization, 2010, 76 (3), pp.600. 10.1016/j.jebo.2010.09.017 . hal-00911819

\section{HAL Id: hal-00911819 https://hal.science/hal-00911819}

Submitted on 30 Nov 2013

HAL is a multi-disciplinary open access archive for the deposit and dissemination of scientific research documents, whether they are published or not. The documents may come from teaching and research institutions in France or abroad, or from public or private research centers.
L'archive ouverte pluridisciplinaire HAL, est destinée au dépôt et à la diffusion de documents scientifiques de niveau recherche, publiés ou non, émanant des établissements d'enseignement et de recherche français ou étrangers, des laboratoires publics ou privés. 


\section{Accepted Manuscript}

Title: A New Test for Chaos and Determinism based on Symbolic Dynamics

Authors: Mariano Matilla-García, Manuel Ruiz Marín

PII: $\quad$ S0167-2681(10)00196-4

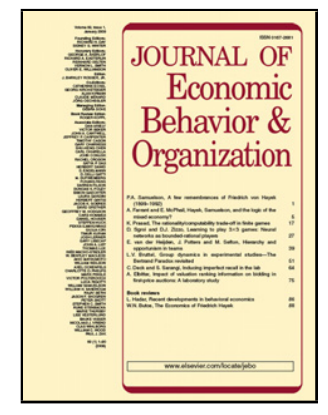

DOI: $\quad$ doi:10.1016/j.jebo.2010.09.017

Reference: $\quad$ JEBO 2623

To appear in: Journal of Economic Behavior \& Organization

Received date: $\quad$ 29-5-2009

Revised date: $\quad 17-9-2010$

Accepted date: $\quad$ 17-9-2010

Please cite this article as: Matilla-García, M., Marín, M.R., A New Test for Chaos and Determinism based on Symbolic Dynamics, Journal of Economic Behavior and Organization (2010), doi:10.1016/j.jebo.2010.09.017

This is a PDF file of an unedited manuscript that has been accepted for publication. As a service to our customers we are providing this early version of the manuscript. The manuscript will undergo copyediting, typesetting, and review of the resulting proof before it is published in its final form. Please note that during the production process errors may be discovered which could affect the content, and all legal disclaimers that apply to the journal pertain. 


\title{
A New Test for Chaos and Determinism based on Symbolic Dynamics.
}

\author{
Mariano Matilla-García*, UNED. \\ Manuel Ruiz Marínł Universidad Politécnica de Cartagena.
}

\begin{abstract}
We propose a novel test to determine, given a time series, if the dynamics are generated by a deterministic (including low dimensional chaos), rather than a stochastic, process. In addition, we introduce a new nonparametric bootstrap test for independence which is consistent against a broad class of alternatives. The conditions under which the tests can be applied are very weak. The advantages of the presented methods are simplicity, invariance with respect to monotonic transformations and the applicability of the tests regardless of the discrete or continuous nature of the data generating process. We conduct several simulation studies to evaluate the performance of our tests on well-known dynamic processes. Finally, our tests are applied to several sets of financial returns that have been recently studied.
\end{abstract}

\section{Introduction}

There has been an important interest in the economic literature in measuring the stability of dynamic systems and, hence, to distinguishing between nonlinear deterministic processes and nonlinear stochastic processes. It is central for economic analysis and economic forecasting to know whether the data at hand are essentially generated by a deterministic or stochastic dynamic system, since the tools available in each case are certainly different. The central role played by nonlinear models in economic modeling is well-established (Granger 2008 and Elliot et al. 2006). A special class of nonlinear models are those that can be grouped under the generic title of chaos. Despite the importance of nonlinear models in both theoretical and forecasting terms, few tests for complex

${ }^{*}$ Author for correspondence. Economía Cuantitativa Aplicada I. Fac. Económicas. Paseo Senda del Rey, 11. CP. 2040. Madrid. Spain. email: mmatilla@cee.uned.es.

${ }^{\dagger}$ Departamento de Métodos Cuantitativos e Informáticos. Calle Real, 3 - 30.201. Cartagena. Spain. 
chaotic (deterministic) processes are available (Granger 2008). In this paper we propose a test for deterministic process versus a stochastic one.

So far, one of the most useful measures of complexity has been the Lyapunov exponent which measures the average rate of divergence or convergence of two nearby trajectories. A positive Lyapunov exponent is generally taken as evidence of the chaotic character of the underlying system. Determinism tests have also existed (see Kaplan and Glass 1992, Yao and Tong 1998 and Binder et al. 2005). Theoretical and empirical research on detection of deterministic-chaotic behavior has disseminated rapidly in the last 30 years. Possible evidence of chaotic behavior has been produced for U.S. business cycle data (Brock and Sayers 1988), weekly stock returns (Scheinkman and LeBaron 1989), Treasury bill returns (Brock and Malliaris 1989), exchange rates returns (BajoRubio et al. 1992), gold and silver returns (Frank and Stengos 1988), energy prices (Matilla-García 2007) among others. Interestingly, results on such series are inconclusive, possibly due to the lack of appropriate testing methods (see Hommes and Manzan 2006 and Gilmore 1993).

Given time series data obtained from an unknown dynamic system, the Lyapunov exponents may be estimated using the phase space reconstruction method of Takens (1981) or by a method based on nonparametric regression, as has been proposed by Eckmann and Ruelle (1985) and Eckmann et al. (1986). These two methodologies are criticized for the absence of a distributional theory providing a statistical framework for hypothesis testing when using the estimated Lyapunov exponents. Other methods like the well-known BDS test (Brock et al. 1996) and the generalized version of the BDS statistic (Matilla-García et al. 2004), both based on the Grasberger and Procaccia (1983) correlation integral, should be viewed as tests for i.i.d. against a general dependence which, among others, include chaos. Therefore they are not testing for chaos. Shintani and Linton (2004) and Fernández-Rogríguez et al. (2005) have developed new techniques in order to give a statistical framework to the Lyapunov exponents methods and, hence, to properly construct a test for chaos.

Shintani and Linton (2004) and Fernández-Rodríguez et al. (2005) correspond, respectively, to the latest improvements of the two main numerical approaches for computing the maximal Lyapunov exponent (namely, the tangent space method and the direct method) ${ }^{1}$. It is well-known, for both approaches, that the output of the Lyapunov-exponent based tests is sensitive to the choice of the parameters embedding dimension and delay (we refer the reader to Casdagli et al. 1991 and

\footnotetext{
${ }^{1}$ Basic references of these methods are McCaffrey et al. (1992), Nychka et al. (1992) or Gençay (1996) and Wolf et al. (1985) Rosenstein et al. (1993) and Kantz (1994).
} 
Schreiber and Kantz 1995 for detailed discussions). Additionally, we note that tangent methods require to decide upon neural network's design.

This paper abandons those methods based on Lyapunov exponents and presents simple new tests for independence and determinism which rely, however, on symbolic dynamics and entropy. Symbolic dynamics studies dynamical systems on the basis of the symbol sequences obtained for a suitable partition of the state space. The basic idea behind symbolic dynamics is to divide the phase space into a finite number of regions and to label each region by an alphabetical symbol. One of the interesting properties of symbolic dynamics is that essential global features of the underlying dynamics, like its deterministic or stochastic nature or its complexity, are kept. Symbolic dynamics has been used for investigation of non-linear and chaotic dynamic systems (for an overview see Hao and Zheng 1998). More precisely, our test relies on the concept of entropy, neither topological entropy nor Kolmogorov-Sinai entropy, but permutation entropy ${ }^{2}$, as defined in Bandt et al. (2002), which is rooted on symbolic dynamics, as will be shown. Entropy has been widely used in econometrics; see Ullah (1996) for a review. Most of this research is related with establishing asymptotic distribution theory for certain entropy measures of serial dependence (Robinson 1991, Granger and Lin 1994, Maasoumi and Racine 2002, Granger et al. 2004 and Hong and White 2005). We use another measure of entropy, permutation entropy, to construct firstly a measure of complexity of a given time series, secondly a bootstrap statistical procedure to test for independence and finally, the basis for the construction of several tests of determinism. In general, entropy is the maximum expected gain of information from knowing an additional point in a typical trajectory of the system. Entropy gives a measure of the uncertainty or volatility of the system under study. In contrast to Lyapunov exponents, that account for sensitivity to the initial conditions as a central characteristic of complex systems, entropy accounts for unpredictably of the system which is another crucial characteristic of complexity. As will be shown, the proposed new approach based on permutation entropy benefits from the property of invariance under any monotonic transformation of the data. Furthermore, its simplicity makes it easy to implement in programming language and hence portability is guaranteed.

In the paper, after presenting the basic new definitions and the theoretical results, the small sample properties of the test are studied using several well-known time series. We also conduct Monte Carlo experiments and we evaluate the performance of the new statistical procedures on deterministic noisy series. Finally, we apply our procedures to the analysis of economic time

\footnotetext{
${ }^{2}$ Interestingly, for certain class of maps, the three entropies coincide.
} 
series that, according to some models, have chaos as a possible source of explanation of certain fluctuations.

The remainder of the paper is organized as follows: Definitions of 'symbol', probability of a 'symbol' and permutation entropy are presented in Section 2, together with an easy example that illustrates these concepts. Section 3 firstly derives a nonparametric test for independence. It is shown that this test is consistent (i.e., it will reject asymptotically with probability one) against the alternative of dependence. We also present a test for determinism that can be easily used together with the previous independence test. Section 4 reports the results of applying our procedure to several well-known numerical time series and we report the results of several Monte Carlo experiments. An empirical application for financial returns is reported in Section 5. Some final remarks are made in the Conclusion.

\section{Definitions and Notation}

The procedure considers ordinal patterns which describe the order relations between some equidistant successive values and then determines the distribution of the ordinal patterns in a time series.

Given a stationary real-valued time series $\left\{x_{t}\right\}_{t \in T}$, ordinal patterns will be defined for a positive integer $m$ usually known as embedding dimension. To that end, the scalar time series is embedded to an $m$-dimensional space: $\mathbf{x}_{m}(t) \equiv\left(x_{t}, x_{t+1}, \ldots, x_{t+(m-1)}\right)$ for $t \in T$.

The ordinal pattern of embedding dimension $m$, at a given time $t$ is defined as the unique permutation $\pi_{m}(t) \equiv\left(\mathrm{r}_{0} \mathrm{r}_{1} \ldots \mathrm{r}_{\mathrm{m}-1}\right)$ of the set $\{0,1, \ldots, m-1\}$ satisfying:

$$
\begin{aligned}
& x_{t+r_{0}} \leq x_{t+r_{1}} \leq \ldots \leq x_{t+r_{m-1}} \\
& r_{s-1}<r_{s} \text { if } x_{t+r_{s-1}}=x_{t+r_{s}}
\end{aligned}
$$

Formula (2) guaranties uniqueness of the permutation defined by (1). This is justified if the values of $x_{t}$ have a continuous distribution so that equal values are very uncommon, with a theoretical probability of occurrence of 0 . It is worth noting that the vector or $m$-history $\mathbf{x}_{t}(m)$ is converted into a unique symbol $\pi_{m}(t)$. Note, as well, that in fact $\pi_{m}(t)$ describes how the order of the dates: $t+0<t+1<\ldots<t+(m-1)$ is turned into the order of the corresponding analyzed values. Consider the following example. Given a time series with seven observations $\{3,6,9,13,5,11,1\}$, according to the previous definition at $t=3$ and for the case of $m=3$, we have $x_{t+2}<x_{t+0}<x_{t+1}$ hence $\pi_{3}(3)=(201)$. 
The main idea is to divide naturally the state space in which the dynamics takes place into a finite number of partitions using the time-dependent information contained in the $m$-history $\mathbf{x}_{m}(t) \in$ $\mathbb{R}^{m}$. Therefore the suggestion is that partitions of the state space are given by comparing neighboring values $x_{t}$. In fact, according to the previous definition, partitions depend on the ordinal structure of the $m$-history. In particular, $\pi_{m}(t)=\pi_{m}(s), s \neq t$ if and only if for all $k, l \in\{0,1, \ldots, m-1\}$ with $k \neq l$ it holds that

$$
x_{t+l}<x_{t+k} \Leftrightarrow x_{s+l}<x_{s+k}
$$

In the above example, $\pi_{3}(3)=\pi_{3}(5)$. In this case the space is divided into 3 ! partitions, because $m=3$, therefore 6 possible permutation symbols $(\pi$ 's) are considered, namely:

$$
\{(012),(021),(102),(120),(201),(210)\}
$$

In general, given a time series $\left\{x_{t}\right\}_{t \in T}$ all $m$ ! permutations of order $m$ are considered here as possible order types of $m$ different numbers. Then the relative frequency of symbol $\pi$ for a given time series and an embedding dimension parameter $m$ can be defined as:

$$
p(\pi)=\frac{\operatorname{card}\left\{t \mid 0 \leq t \leq T-(m-1), \mathbf{x}_{m}(t) \text { has type } \pi\right\}}{T-(m-1)}
$$

For finite series of values the relative frequency (3) is easily calculated. Consider the example given above for $m=3$. The five triplets of possible neighbors are organized in accordance with permutation symbols. Hence $(3,6,9),(6,9,13)$ is represented by permutation $(012),(9,13,5)$ corresponds to permutation $(201),(13,5,11)$ to $(120)$ and $(5,11,1)$ again to $(201)$. Therefore the relative frequency of each symbol is: $p((012))=\frac{2}{5} ; p((201))=\frac{2}{5} ; p((120))=\frac{1}{5}$, while $p((021))=p((102))=p((210))=0$

Naturally, (3) depends also on $m$, therefore it might be studied for various $m s$. Particularly, for $m=2$, there exist two possible permutations, namely, (01) and (10). We find in the example four and two pairs for each permutation, respectively. Therefore, in this case we have $p((01))=\frac{4}{6}$ and $p((10))=\frac{2}{6}$.

We now use the information obtained from the permutation symbols of a given time series to group sample data according to $m$ ! mutually different order patterns, i.e. to classify it into different fixed categories. As shown before, (3) provides a natural method for computing a frequency distribution for each permutation symbol. Notice that we are not interested in ordinal patterns (found in a given time series) themselves, but in their distribution. In other words, ordinal patterns are each identified with exactly one of the permutation symbols and according to (3), we are 
concerned about the relative frequencies of the symbols. In this fashion, the main point is to summarize the original time series into a set of relative frequencies, and then to use this set for conducting a study based on information theory.

In general, complexity measures have been developed in order to distinguish regular, chaotic and random behavior. Among these measures, those based on information theory consist of calculating several entropies (see Bandt et al. 2002). We focus on the entropy contained in partition of the phase-space given by all the possible symbols $(\pi$ 's).

Let us consider a symbol as a random vector $\pi=\left(\mathrm{r}_{0} \mathrm{r}_{1} \ldots \mathrm{r}_{\mathrm{m}-1}\right) \in S_{m}$, where $S_{m}$ denotes the symmetric group of order $m$ !, that is the group formed by all the permutations of length $m$. (3) assigns the $p\left(\pi=\pi_{i}\right)$ to each symbol of the symmetric group, satisfying that $\sum_{i=1}^{m !} p\left(\pi_{i}\right)=1$. A measure of information content from observations in $p(\pi)$ is $\log (p(\pi))^{-1}=-\log p(\pi)$ and the expected information $(E)$ is given by

$$
h(m) \equiv-E \log p(\pi)=-\sum_{i=1}^{m !} p\left(\pi_{i}\right) \log p\left(\pi_{i}\right)
$$

we refer to this measure of information as permutation entropy, since it stands for Shannon' entropy (1948) for $m$ ! distinct symbols.

The most interesting properties of entropy as an information measure (see Ullah 1996) are shared by (4). Lack of information, ie. $-\log p\left(\pi_{i}\right)=0$, is obtained when the event $\pi=\pi_{i}$ occurs with complete certainty, that is when the $p\left(\pi_{i}\right)=1$ and $p\left(\pi_{j}\right)=0$ for every $j \neq i$. Similarly, a high degree of information is achieved when the event $\pi=\pi_{i}$ occurs with $p\left(\pi_{i}\right)$ tending to zero for all $i$, since $-\log p\left(\pi_{i}\right)$ tends to $+\infty$. As an information measure, (4) is then a measure of uncertainty, disorder or volatility associated with a symbol variable.

Following with the previous example, the permutation entropy is easily computed by $h(m=$ $3)=-\sum_{i=1}^{3 !} p\left(\pi_{i}\right) \log p\left(\pi_{i}\right)=0.45$.

An interesting property of permutation entropy is that ordinal patterns clearly do not change under monotonic transformations, and this nice property means that ordinal patterns, and therefore (4) for a given $m$, are invariant with respect to different monotonic transformations of the original time series. Invariance is important since otherwise clever transformations would produce different levels of uncertainty.

It is important to note that the researcher has to decide upon the embedding dimension $m$ in order to compute permutation entropy. Fortunately, this decision can be easily conducted. Note that $T$ should be larger than the number of permutation symbols $m$ ! in order to have at least 
the same number of $m$-histories as possible symbols (events) $\pi_{i}, i=1, \ldots, m !$. However, for practical proposes, we require to work with data sets containing at least five times the number of possible events ${ }^{3}$. For instance, a data set of 200 observations is enough for computing $h(4)$ because 24 symbols are obtained for $m=4$ (in fact, a set of 120 would be a sufficient amount of data); similarly, 600 observations is the smallest data set that can be considered for an embedding dimension of 5 since in this case 120 symbols might be found. Beyond embedding dimension of 6 , data requirements are unrealistic for real economic time series, so we do not use such dimensions. Through this paper we compute permutation entropy in a manner that the researcher has not to choose the embedding dimension: For a given data set of $T$ observations, the embedding dimension will be the largest $m$ that satisfies $5 m ! \leq T$ with $m=2,3,4, \ldots$. For example, for case of $T=500$, we $\operatorname{set}^{4} m=4$.

\section{Testing for determinism with permutation entropy}

Distinguishing between deterministic chaos and stochastic randomness has been challenging so far in the relevant literature (see among others Kaplan and Glass 1992, Yao and Tong 1998, Gottwald and Melbourne 2004, 2005, Binder et al. 2005 and Granger 2008). Practical consequences are derived from a correct identification diagnostic about the nature of the observed or experimental time series. A spurious identification will lead, when dealing with economic time series, to poor results, since modeling and forecasting will be conducted and designed differently depending on whether the data at hand are essentially generated by a deterministic system or by a stochastic dynamic system.

In this section we present several procedures, but only one of them is a test for determinism. All the procedures introduced are based on symbolic dynamics (particularly on permutation entropy). In the first subsection we give (i) a complexity measure and, based on it, we show that (ii) a nonparametric and consistent test for independence can be easily obtained. In the second subsection, we show how it is possible to construct (iii) a test that detects deterministic (both chaotic and

\footnotetext{
${ }^{3}$ Other statistical techniques that, as ours, are based on mutually exclusive categories require a sample size (T) enough to expect a frequency $\geq 5$ for each symbol (see chapter 10 of Rohatgi 1976). For this reason, we require to work with data sets containing at least five times the number of possible events (symbols).

${ }^{4}$ Note that if $T$ is large $(T>25.200)$, then the selected $m$ will be large as well (indeed $\left.m>7\right)$, and hence the procedure will be too expensive in terms of computational time. For this reason, and because of the usual length of economic time series, we recommend to operate with $m=6$ for $T>3600$.
} 
regular) dynamics based on a new general feature (ie., an intrinsic property) that characterizes non-stochastic and stochastic processes, and hence it allows to distinguish between deterministic time series and stochastic time series. Advantages of procedures (i),(ii) and (iii) are discussed in other sections.

\subsection{A 0-1 measure for complexity and a bootstrap permutation entropy based test for independence}

We firstly propose a new procedure for measuring the complexity of given time series. In this first new procedure, the input is the time series and the output is a number within the unit interval $[0,1]$ (which serves us to name the technique) ${ }^{5}$. Hence, for every unknown dynamical process, either continuous or discrete, the new method will assign a single number that will serve as a base for initially hinting the nature (random or deterministic) of the underlying system. Naturally, the concept of noisy-chaos (or operational determinism) is a border case whose nature lead us to a semantic (if not philosophical) implications which are beyond the scope of this paper ${ }^{6}$. We focus basically on discriminating low-dimensional deterministic systems from stochastic ones.

As stated in the introduction and differently from other approaches, our method does not rely on a maximal Lyapunov exponent; rather, it relies on the concept of permutation entropy given in the preceding section. Particularly, it is based on the fact that permutation entropy $h(m)$ is bounded: $0 \leq h(m) \leq \log (m !)$. The upper bound is attained for a completely independent random sequence, where all symbols appear with the same probability, i.e. $\frac{1}{m !}$. The lower bound is reached for increasing or decreasing sequence of values. Hence, the time series under study presents some sort of dynamics when $h(m)<\log m$ !.

Note that in order to exactly determine $h(m)$ we first need to assume that the time series is infinite, $\left\{x_{t}\right\}_{t=1}^{\infty}$. Then it is possible to verify that $\lim _{t \rightarrow \infty} p\left(\pi_{i}\right)$ exists when the following very weak stationary condition is fulfilled: for $k \leq m$, the probability of $x_{t}<x_{t+k}$ does not depend on $t$ (see Bandt et al., 2002).

\footnotetext{
${ }^{5}$ Gottwald and Melbourne 2004, 2005 have introduced a 0-1 procedure for detecting chaotic dynamics versus nonchaotic ones. Differently from our procedure, their techniques are binary and do not allow for quantifying the complexity of the system.

${ }^{6}$ However, we empirically treat the case of noisy chaos through out some examples in this paper, but not in this section where we only put forward the new technique.
} 
From the bounded relation we construct the ratio:

$$
H(m)=\frac{h(m)}{\log (m !)}
$$

Obviously, $0 \leq H(m) \leq 1$. If the dynamic process that generated the time series $\left\{x_{t}\right\}$ is deterministic (including regular and chaotic behavior), then with probability one $H(m)$ is 'clearly' distant from 1. However, if the time series $\left\{x_{t}\right\}$ is generated from a purely stochastic serially independent process, then asymptotically $H(m)$ will be 1 . The output of the procedure can be understood as a quantity to measure the presence of nonlinear dynamics (deterministic or random). We emphasize that the procedure based on $H(m)$ cannot be used directly to distinguish between a deterministic and a stochastic but serially dependent process, since in both cases the measure will take values less than unity. Notice as well that, theoretically, for a deterministic low dimensional process, $H(m)$ will take values less than those obtained for a stochastic serially dependent one, and hence it can be of some help to detect determinism. On the contrary, it can be clearly utilized to construct a test for serial (linear or nonlinear) dependence, as we show below.

A consequence of the bounded behavior of $H(m)$ is that it can be used as a base for developing a test for independence. Bootstraps methods are an ideal tool to testing for independence (Skaug and Tjøstheim 1996). Hence, by using bootstraps, we shall test the following null hypothesis:

$$
H_{0}:\left\{X_{t}\right\}_{t \in I} \quad \text { Independent }
$$

against any other alternative. We denote by $\hat{H}(m)$ the estimator of $H(m)$. Therefore, it is natural to compute the bootstrap $p$-value of the quantity $H(m)$ by resampling the original data several times $^{7}$, calculating the corresponding set of $\hat{H}(m)$ s, and then comparing the value $\hat{H}(m)$ from the original data with those found in bootstrap replications. The null is rejected when the observed $\hat{H}(m)$ is among the top $P-\%$ of the bootstrapped values, where $P$ is the confidence level. Moreover with this procedure the test will be consistent. Indeed, let $N$ be the number of bootstrap of the time series $\left\{X_{t}\right\}_{t \in I}$ and denote by $H^{b_{i}}(m)$ the value of $H(m)$ in the $i$-th bootstrap of the series. Now define the following indicator function

$$
\mathcal{I}_{i}= \begin{cases}0 & \text { if } H^{b_{i}}(m)<\hat{H}(m) \\ 1 & \text { if } H^{b_{i}}(m) \geq \hat{H}(m)\end{cases}
$$

Now with the notation introduced above we can state next result.

\footnotetext{
${ }^{7}$ In this paper, whenever the independence test is computed, the number of resamples are 500.
} 
Proposition 1 Let $\left\{X_{t}\right\}_{t \in I}$ be a stationary time series with $|I|=T$ and $m \in \mathbb{N}$ with $m \geq 2$. Assume that the series $\left\{X_{t}\right\}_{t \in I}$ is serially dependent of order $\leq m$. Then,

$$
\lim _{T \rightarrow \infty} \operatorname{Pr}\left(\sum_{i=1}^{N} \mathcal{I}_{i}=N\right)=1
$$

Proof. Firstly note that the estimator $\hat{H}(m)=\frac{-\sum_{i=1}^{m !} \hat{p}\left(\pi_{i}\right) \log \hat{p}\left(\pi_{i}\right)}{\log (m !)}$ is consistently estimated because $p \lim _{T \rightarrow \infty} \hat{p}\left(\pi_{i}\right)=p\left(\pi_{i}\right)$ (see Bandt et al. 2002) and hence $p \lim _{T \rightarrow \infty} \hat{H}(m)=H(m)$. Notice as well that if $T \rightarrow \infty$, then the bootstrap generates an independent series and therefore $H^{b_{i}}(m) \simeq 1$ for all $i=1,2 \ldots, N$. On the other hand, since the original series $\left\{X_{t}\right\}_{t \in I}$ is, by assumption, serially dependent of order $\leq m(H(m)<1)$, we have that $\lim _{T \rightarrow \infty} \operatorname{Pr}\left[\left(H^{b_{i}}(m)-H(m)\right)>0\right]=1$ and hence the result follows.

This is a valuable property since the test will reject asymptotically serial independence whenever there is serial dependence alternatives of order $\leq m$. We remark that the required assumptions for applying the entropy based test for independence are really mild, as compare with other tests in the literature, which importantly facilitates its use and makes it relatively valuable.

For illustrative purposes we compare the output of the 0-1 measure for complexity and the output of the bootstrap test for independence on both random and purely deterministic sequences. The Logistic map, together with Henon and Lorenz models, are well-known chaotic systems. We have also included (jointly with an iid standard Normal) an iid Beta since its distribution function is similar to the one generated by logistic map. The models are the following:

(i) The logistic chaotic model:

$$
y_{t}=4 y_{t-1}\left(1-y_{t-1}\right) \text { with } y_{0}=0.7
$$

(ii) The Henon system:

$$
\left.\begin{array}{rl}
x_{t} & =1+y_{t-1}-1.4 x_{t-1}^{2} \\
y_{t} & =0.3 x_{t-1} \\
\text { with } & x_{0}=1 \text { and } y_{0}=0 .
\end{array}\right\}
$$

(iii) The Lorenz system:

$$
\left.\begin{array}{rl}
\dot{x} & =-16(x-y) \\
\dot{y} & =-x z+45.92 x-y \\
\dot{z} & =x y-4 z
\end{array}\right\}
$$


with $x(0)=0.2, y(0)=0.4$ and $z(0)=20$.

(iv) The iid normal distribution $y_{t}=\varepsilon_{t}$ with $\varepsilon_{t} \sim N(0,1)$

(v) The Beta distribution $B(0.5,0.5)$.

For each model, three sample sizes have been chosen: $\mathrm{T}=3600, \mathrm{~T}=600$ and $\mathrm{T}=120$. In order to compute the test we have set $m=6, m=5$ and $m=4$ for each sample size, respectively.

Table 1. 0-1 measure for complexity and the bootstrap test for independence

\begin{tabular}{cccc} 
Models & $H(6)$ & $H(5)$ & $H(4)$ \\
\hline Logistic & 0.4303 & 0.4410 & 0.4634
\end{tabular}

\begin{tabular}{cccc} 
HENON & 0.5521 & 0.6047 & 0.6748 \\
LoRENZ & $(0)$ & $(0)$ & $(0)$ \\
& 0.1435 & 0.1836 & 0.2482 \\
$\operatorname{BETA}(0,5 ; 0,5)$ & 0.9892 & 0.9838 & 0.9881 \\
$\mathrm{~N}(0,1)$ & $(0.45)$ & $(0.41)$ & $(0.64)$ \\
& 0.9825 & 0.9818 & 0.9846 \\
& $(0.43)$ & $(0.54)$ & $(0.55)$ \\
\hline
\end{tabular}

$H \approx 1$ for random finite sequences. $0<H<<1$ for deterministic sequences.

Bootstrap p-values under the null of independence are given in parenthesis.

It is clear from Table 1 that for models (i) to (iii), the 0-1 procedure yields a 'measure' clearly below one, while the output is close to one for models (iv) and (v), as expected by the theory. On the other hand, regarding the entropy based test for independence, it can be observed that the null is rejected for models (i) to (iii), while the null cannot be rejected for the remainder cases (as expected as well).

It is remarkable and useful in practice the fact that both, the test for independence and the 0-1 procedure, can be easily used together in order to obtain relevant information about some properties of the underlying system.

Despite the fact that $H(m)$ takes values clearly less than unity for a deterministic process, while it takes values close to 1 for stochastic serially dependent ones, from a practical point of view, it 
is advisable to evaluate whether two outputs of the 0-1 measure are significantly different. This problem is especially relevant when the outputs are 'close' to 1 which usually occurs for stochastic serial dependent processes. In order to deal with this issue, in the next section, we properly construct a statistical test for determinism that can also be used together with the bootstrap test for independence and with $0-1$ procedure. Through out the rest of the paper we utilize these techniques, and hence utility of the presented tools can be clearly evaluated.

\subsection{A Test for Determinism}

The purpose of this subsection is to elaborate a test that allows to discriminate between deterministic and stochastic (serially or non-serially dependent) processes. In order to construct a feasible test for determinism we focus on our previous findings regarding the concept of permutation entropy. For a finite amount of data $(T<\infty)$ we study, given an embedding dimension $m$, the behavior of $h(m)$ when increasing the number of possible observable symbols.

Fix $w, k \in \mathbb{N}$ such that $w=\frac{m !}{k}$ and $w<<m$ !. Let

$$
\mathcal{W}_{1} \subseteq \mathcal{W}_{2} \subseteq \cdots \subseteq \mathcal{W}_{k}
$$

be $k$ subsets of the symmetric group of order $m !\left(S_{m}\right)$. The sets $\mathcal{W}_{j}$ are constructed recursively as follows: $\mathcal{W}_{1}$ is a set of $w$ symbols chosen at random in $S_{m}$. Then

$$
\mathcal{W}_{j}=\mathcal{W}_{j-1} \cup\left\{w \text { symbols chosen at random in } S_{m} \backslash \mathcal{W}_{j-1}\right\}
$$

for $j=2, \ldots, k$.

We define the next modified permutation entropy's function

$$
h^{\mathcal{W}_{j}}(m)=-\sum_{\pi_{i} \in \mathcal{W}_{j}} p\left(\pi_{i}\right) \log p\left(\pi_{i}\right)
$$

Now, (4) can be understood as the limit behavior of $h^{\mathcal{W}}(m)$ since, as can be easily verified, it holds that:

$$
h(m)=\lim _{j \rightarrow k} h^{\mathcal{W}_{j}}(m)
$$

We are not interested in $h(m)$ itself, but in the sequence $\left\{h^{\mathcal{W}_{j}}(m)\right\}_{j=1}^{k}$ which contains $k=m ! / w$ points.

Theorem 1 Let $\left\{X_{t}\right\}_{t \in I}$ be a real stationary time series and $m$ a fixed embedding dimension. Then one of the following conditions holds: 
1. $\left\{X_{t}\right\}_{t \in I}$ is non-deterministic (i.e., stochastic) and $h^{\mathcal{W}_{j}}(m)<h^{\mathcal{W}_{j+1}}(m)$ for all $j=1,2, \ldots, k$.

2. $\left\{X_{t}\right\}_{t \in I}$ is deterministic and there exists $j_{0}$ such that $h^{\mathcal{W}_{j_{0}}}(m)=h^{\mathcal{W}_{j}}(m)$ for all $j_{0} \leq j \leq k$.

Proof. If the underlying process is non-deterministic (i.e., serially or non-serially stochastic dependent process), the maximum gain of information $\left(h^{M_{j}}(m)\right)$ is reached only when $h^{\mathcal{W}_{j}}(m)$ reaches the limit point given by (9). More concretely, for any subset $K \subseteq S_{m}$ it follows that $p(K)<p(K \cup\{\pi\})$ for every $\pi \in S_{m} \backslash K$. Therefore

$$
-\sum_{\sigma \in K} p(\sigma) \log (p(\sigma))<-\sum_{\sigma \in K \cup\{\pi\}} p(\sigma) \log (p(\sigma))
$$

for every $\pi \in S_{m}$ and hence it holds that $h^{\mathcal{W}_{j+1}}(m)-h^{\mathcal{W}_{j}}(m)>0$ for $j=1,2, \ldots, k-1$. However, if the process is chaotic (deterministic), the maximum gain of information $h^{M_{j}}(m)(<<\log (m !))$ is reached much before achieving the limit point (9). Indeed, there exists a subset $H \subseteq S_{m}$ such that $p(H)=p(H \cup\{\pi\})$ for every $\pi \in S_{m}$. Hence

$$
-\sum_{\sigma \in H} p(\sigma) \log (p(\sigma))=-\sum_{\sigma \in H \cup\{\pi\}} p(\sigma) \log (p(\sigma))
$$

for every $\pi \in S_{m}$. Therefore there exists $1 \leq j_{0} \leq k$ such that $h^{\mathcal{W}_{j_{0}+s}}(m)=h^{\mathcal{W}_{j_{0}}}(m)$ for every $0 \leq s \leq k-j_{0}$ and thus the increasing behavior of $h^{\mathcal{W}_{j}}(m)$ does not hold. In fact $h^{\mathcal{W}_{j}}(m)$ reaches its limit point several times, not only once as it happens in the stochastic case.

This theorem shows a new attribute that necessarily distinguishes any deterministic (chaotic or regular) process from a non-deterministic one. Intuitively, the theorem puts forward that the information (or complexity) measured via permutation entropy reaches a level such that, in case of deterministic dynamics, no more information is captured by increasing the symbols under consideration. In other words, the level of information obtained from $h(m)$ by including new symbols saturates (i.e., reaches its maximum) clearly below from the level of complexity that would be found in case of a process of nondeterministic nature.

Notice that according to Theorem 1 and taking into account that $h^{\mathcal{W}_{j}}(m) \leq \log (j w)$, if the process is stochastic, the sequence $\left(h^{\mathcal{W}} \mathcal{W}_{j}(m)\right)_{j}$ will scale with $(\log (j w))_{j}$, while this will not occur if the process is deterministic (chaotic or regular). As an example, observe that panels (a) and (b) of Fig. 1 show how the scaling behavior of the standard normal random process, contrasts with the absence of such behavior in the deterministic Logistic map ${ }^{8}$.

\footnotetext{
${ }^{8}$ All calculations in Fig. 1 have been done for $T=3600$.
} 

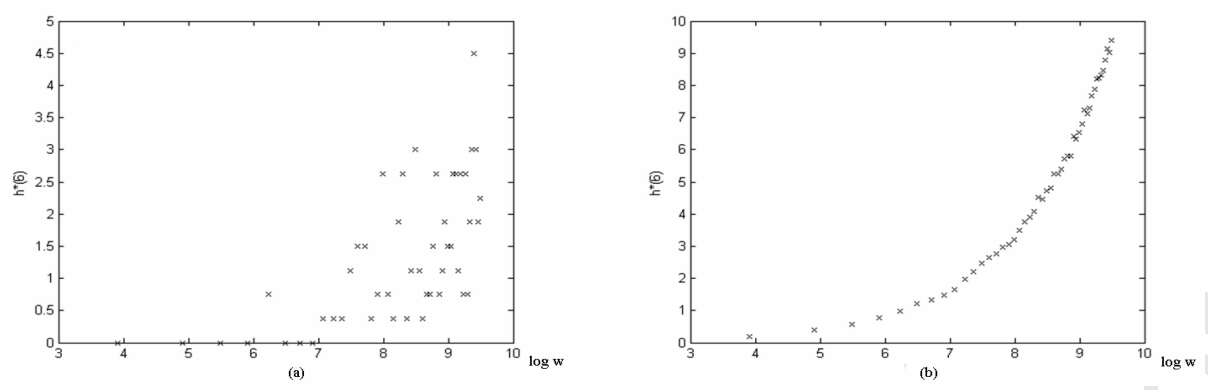

Figure 1: Scaling behavior of the modified $h^{\mathcal{W}_{j}}(m)$ for the Logistic Map (a) and for the standard Normal distribution (b).

A new feature that makes a distinction between deterministic and nondeterministic time series has been put forward. This new attribute it is now used to construct a test for determinism.

Let $d h^{\mathcal{W}_{j}}(m)$ denotes the numerical slope of $h^{\mathcal{W}_{j}}(m)$, that is

$$
d h^{\mathcal{W}_{j}}(m)=\frac{h^{\mathcal{W}_{j+1}}(m)-h^{\mathcal{W}_{j}}(m)}{\log \frac{j+1}{j}}
$$

According to Theorem 1 and its scaling behaviour (see also Fig. 1), the numerical slope of permutation entropy of a random process will increase with $(\log (j w))$, while this will not hold for chaotic or regular processes (see for instance panel $a$ for the Logistic map data set) for which the $d h^{\mathcal{W}_{j}}(m)$ varies in a non-increasing way. This property of the numerical slope of $h^{\mathcal{W}}{ }_{j}(m)$ can be tested using a classical econometric test by performing the following regression:

$$
d h^{\mathcal{W}_{j}}(m)=\alpha_{0}+\alpha_{1} j+\varepsilon_{j}, \quad \text { for } \quad j=1,2, \ldots k-1
$$

where $\varepsilon_{j}$ is independent white noise with $E\left(\varepsilon_{j}^{2}\right)=\sigma^{2}$ and $E\left(\varepsilon_{j}^{4}\right)<\infty$. According to definition of $h^{\mathcal{W}_{j}}(m)$, regression (10) relates the average gain of information due to the evaluation of a random set of symbols with the number of symbols. By Theorem 1, regression (10) will capture the fact there exists $j_{0}$ such that beyond $j_{0}$ the $d h^{\mathcal{W}_{j}}(m)$ does not increase under the null of determinism, while the opposite will happen under the alternative hypothesis (ie., stochastic process). On the other hand, regression (10) can be understood as a simple symbol-trend model. As in the wellknown simple time-trend model, the OLS estimates $\hat{\alpha}_{0}$ and $\hat{\alpha}_{1}$ are so that asymptotically the usual t-test of $\mathrm{H}_{0}$ is valid ${ }^{9}$. As a result, the estimated parameter $\hat{\alpha}_{1}$ can be used to test that $d h^{\mathcal{W}}{ }_{j}(m)$ does not increase with $j$, which implies an underlying deterministic process.

\footnotetext{
${ }^{9}$ See, for example, the proof given in Hamilton (1994), pags. 454-463.
} 
The null hypothesis and the alternative hypothesis are then expressed as follows ${ }^{10}$ :

$$
\begin{aligned}
& H_{0}: \alpha_{1}=0 \text { (deterministic process) } \\
& H_{1}: \alpha_{1}>0 \text { (stochastic process) }
\end{aligned}
$$

Table 2. Outputs of the Test For Determinism for Deterministic and Stochastic realizations.

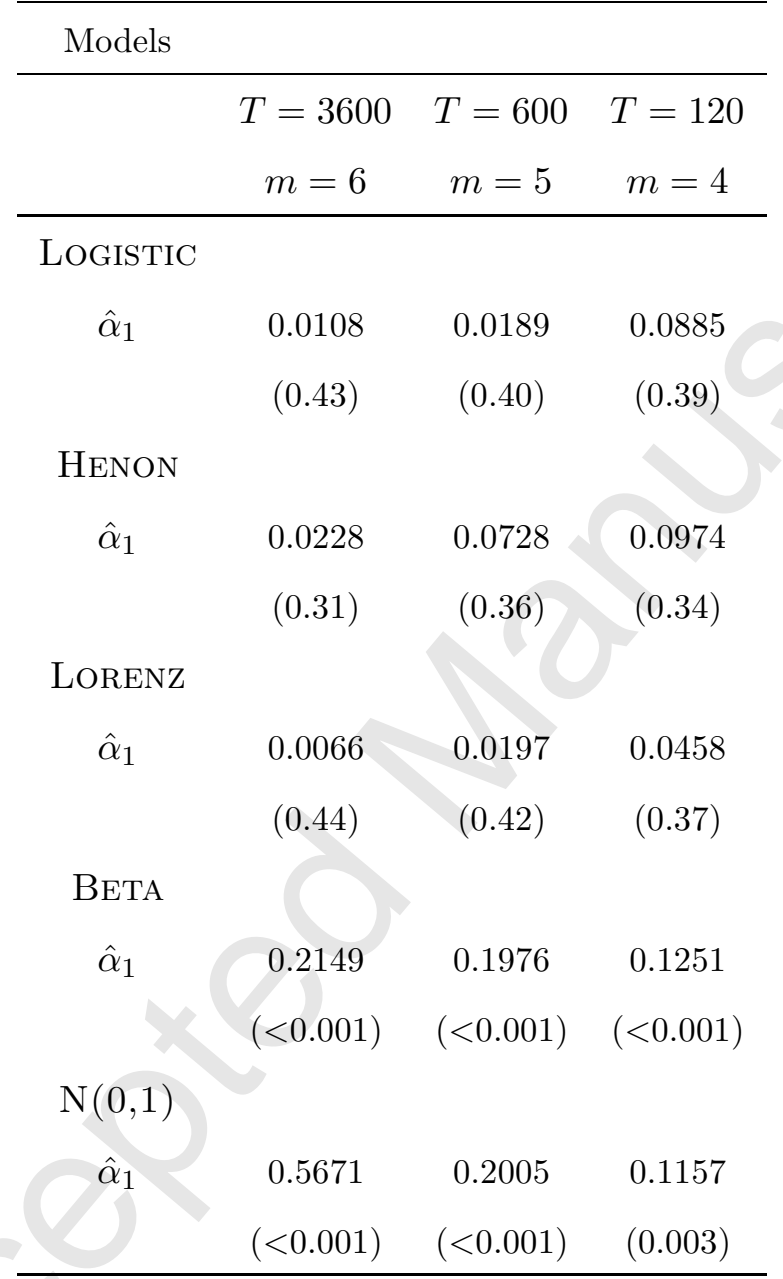

Note: p-values for the null of determinism are in parenthesis. Last column is calculated with $w=1$.

In order to calculate the test $(10)$ it is necessary to select $w \in \mathbb{N}$. In our calculations we have generally set $w=5$ which, for a given $m$, implies $k=m ! / 5$. Other $w$ s can be selected with the condition that $w<<m$ ! and hence to obtain a significant number of $h^{\mathcal{W}_{j}}(m)$ to estimate (10).

Results of the two proposed tests are reported in Table 2. As expected, the null of determinism is not rejected for the three deterministic processes, regardless of the sample size. Non-significant slopes are found for these systems. On the contrary, the null of determinism is rejected, in favor of randomness, for standard Normal and Beta realizations since numerical slopes $\left(d h^{\mathcal{W}_{j}}(m)\right)$ are significantly different from zero.

\footnotetext{
${ }^{10}$ Notice that the test is one-sided since $\hat{\alpha}_{1}$ cannot be negative by construction of the test.
} 


\section{Numerical results}

In this section we firstly focus on studying the properties of the $0-1$ measure for complexity, the bootstrap test for independence, and the test for determinism when dealing with time series that have been traditionally well analyzed with other statistical procedures. Secondly, we concentrate on conducting Monte Carlo experiments to explore the behavior of the tests on finite samples. And finally, we use our techniques for analyzing time series generated from a chaotic financial economic model which is contaminated with noise. In addition we also include a comparison with Shintany and Linton's test for chaos.

We have considered a relatively small sample size (realistic for economic time series) of 380 data points and another of 2000 observations as it is of interest to examine the performance of our statistical procedures when modifying the sample size.

Our first set of numerical results evaluates six models that are collected in Table 3. The first model is the logistic map with dynamic noise ${ }^{11}$ :

$$
\text { (Model 1) } y_{t}=a y_{t-1}\left(1-y_{t-1}\right)+\sigma \varepsilon_{t}
$$

where $\varepsilon_{t} / v_{t} \sim U(-1,1)$ independent of $y_{t}$, and $^{12}$

$$
v_{t}=\min \left\{a y_{t-1}\left(1-y_{t-1}\right), 1-a y_{t-1}\left(1-y_{t-1}\right)\right\}
$$

This simple one-dimensional map develops complex behavior depending on the parameter $a$. Interestingly, for $0<a<3$, the map is globally stable (which implies a negative Lyapunov exponent when $\sigma=0$ ) and it is chaotic for $3.57<a \leq 4$ (positive Lyapunov exponent when $\sigma=0$ ). The results are based on the parameters $a=4, \sigma=0.25$, with the two sample sizes already mentioned. We have also simulated the logistic system in the stable case of $a=1.5$.

The other five models come from a crucial paper that aimed to distinguish between non-linear deterministic processes and non-linear stochastic processes in the singled-blind controlled competition conducted by Barnett et al. (1997). Powerful properties of those methods based on Lyapunov exponents were highlighted. It is of interest to examine how our new statistical procedures perform for the same data sets. The competition ${ }^{13}$ used the same sample sizes that we are considering.

\footnotetext{
${ }^{11}$ The noise is introduced as in Shintani and Linton(2004) to help further comparisons.

${ }^{12}$ The following expression ensures that the process $y_{t}$ is restricted to the unit interval.

${ }^{13}$ The data is downloaded from the archive given in Barnett et al. (1997), footnote 2.
} 
Both, the small sample and the large sample, were obtained from a single observation generated from the following five models, where $u_{t}$ is an i.i.d. standard normal random variable.

(Model 2) Logistic map

$$
y_{t}=3.57 y_{t-1}\left(1-y_{t-1}\right) \text { with } y_{0}=0.7
$$

(Model 3) A GARCH process of the form

$$
y_{t}=h_{t}^{1 / 2} u_{t} \quad h_{t}=1+0.1 y_{t-1}^{2}+0.8 h_{t-1} \quad \text { with } y_{0}=0 \text { and } h_{0}=1
$$

(Model 4) A non-linear moving average (NLMA) process

$$
y_{t}=u_{t}+0.8 u_{t-1} u_{t-2}
$$

(Model 5) An ARCH process of the form

$$
y_{t}=\left(1+0.5 y_{t-1}^{2}\right)^{1 / 2} u_{t} \text { with } y_{0}=0
$$

(Model 6) An ARMA process of the form

$$
y_{t}=0.8 y_{t-1}+0.15 y_{t-2}+u_{t}+0.3 u_{t-1} \text { with } y_{0}=1 \text { and } y_{1}=0.7 \text {. }
$$

Notice that Model 2 is purely deterministic, while Models 3-6 are stochastic. 
Table 3 0-1 measure, Test for Independence and Test for Determinism.

\begin{tabular}{|c|c|c|c|c|c|}
\hline Process & $T=2000$ & $T=380$ & Process & $T=2000$ & $T=380$ \\
\hline Deterministic noise & & & NLMA & & \\
\hline $\mathrm{H}$ & 0.10 & 0.21 & & 0.99 & 0.98 \\
\hline pboots.-value & $(0.00) \quad(0.00)$ & $(0.00) \quad(0.00)$ & & $(0.00)$ & $(0.00)$ \\
\hline \multirow[t]{2}{*}{$\hat{\alpha}_{1}$} & $0.12 \quad 0.01$ & $0.13 \quad 0.02$ & & 0.47 & 0.26 \\
\hline & $(0.24) \quad(0.15)$ & $(0.15)$ & & $(<0.001)$ & $(<0.001)$ \\
\hline \multicolumn{6}{|l|}{ Logistic Map } \\
\hline $\mathrm{H}$ & 0.43 & 0.45 & & 0.99 & 0.99 \\
\hline pboots.-value & $(0.00)$ & $(0.00)$ & & $(0.03)$ & $(0.71)$ \\
\hline \multirow[t]{2}{*}{$\hat{\alpha}_{1}$} & 0.03 & 0.01 & & 0.60 & 0.27 \\
\hline & $(0.48)$ & $(0.47)$ & & $(<0.001)$ & $(<0.001)$ \\
\hline \multicolumn{6}{|l|}{ GARCH } \\
\hline $\mathrm{H}$ & 0.99 & 0.99 & & 0.90 & 0.90 \\
\hline pboots.-value & $(0.60)$ & $(0.38)$ & & $(0.00)$ & $(0.00)$ \\
\hline \multirow[t]{2}{*}{$\hat{\alpha}_{1}$} & 0.57 & 0.17 & & 0.18 & 0.20 \\
\hline & $(<0.001)$ & $(<0.001)$ & & $(0.005)$ & $(0.03)$ \\
\hline
\end{tabular}

Note: The 0-1 procedure $(\mathrm{H})$, bootstrap independence test, and test for Determinism $\left(\hat{\alpha}_{1}\right)$ have been computed for $m=5$ if $T=2000$, and $m=4$ if $T=380$. pboots.-values refer to the p-value under the null of independence.

Results for the two processes generated from Model 1 are given in Table 3 under the label of 'Deterministic noise'. The italics are reserved for results of the stable case. The 0-1 measures are clearly below 1 and their corresponding bootstrap $p$-values under the null of independence are zero. These results indicates that the $0-1$ procedure jointly with the bootstrap independence test have clearly detected the dependence structure of the generating mechanism. Importantly, the detected dependence can be now classified by means of our test for determinism. The p-values under the null of determinism suggest not rejecting the null, pointing to the deterministic skeleton of the studied process.

The results for the data sets generated from Models 2-6 can be summarized as follows. Regarding the logistic data set, the 0-1 procedure, together with the test for independence, detects that the process contains structure and, according to the results for the tests for determinism, such dependence is of deterministic nature. As regards purely stochastic time series (GARCH, NLMA, ARCH and ARMA), several interesting observations can be offered. Firstly, we note that the 0-1 
measures are close to 1 and that the test for determinism rejects the null, suggesting the stochastic nature of the underlying generating processes. Secondly, it can be observed that for the NLMA data set the $0-1$ measure is almost 1 , while the p-value of the bootstrap test for independence suggests a rejection of the null; in other words, the complexity detected by $0-1$ procedure is not due to an independent process, but due to possibly nonlinear stochastic dynamics. The same occurs for the $\mathrm{ARCH}$ data set, but only for the larger data set where dependence is detected. On the contrary, the results for the GARCH data set will lead the researcher to wrongly not rejecting independence.

These results (a) concur with the conclusions given in Shintani and Linton (2004), and (b) outperform those found with other statistical techniques (see Barnett el al., 1997; Matilla-García et al., 2004; and Fernández-Rodríguez et al. 2005). In consequence, validity and utility of our testing procedures seems to be confirmed.

In the second set of numerical analysis section, we empirically compute the size and the power of our statistical tests, for relatively small sample sizes (namely, $T=380$ and $T=2000$ ). To this end we consider stochastic models (Models 2-6: GARCH, NLMA, ARCH and ARMA) as well as deterministic ones given in section 3 (ie., Logistic, Henon and Lorenz). For each model, we first generate $T+200$ observations and then discard the first 200 to mitigate the impact of initial values. Finally we compute the percentage of rejections of the null using significance levels of 5 per cent. Notice that the null hypothesis with the bootstrap independence test is independence, while for the other test the null is determinism.

As we can see in Table 4, in general terms, the power of the independence test is especially relevant in all cases with the exception of the $\mathrm{ARCH}$ and $\mathrm{GARCH}$ processes. However, by increasing the sample size the problem seems to disappear. Regarding the results of the new test for determinism, it emphatically rejects the null for stochastic processes and adequately accepts the deterministic nature of the chaotic processes. 
Table 4. Monte Carlo results for the Test for Independence and the Test for Determinism

\begin{tabular}{|c|c|c|}
\hline & \multicolumn{2}{|c|}{$T_{1}=380\left(T_{2}=2000\right)$} \\
\hline & $H-$ independence test & $\alpha_{1}-t e s t$ \\
\hline & $\operatorname{Pr}($ reject independence/non-independent) & $\operatorname{Pr}($ reject determinism/stochastic model $)$ \\
\hline GARCH & $10.2(62.3)$ & $100(100)$ \\
\hline NLMA & $99.7(100)$ & $99.0(100)$ \\
\hline $\mathrm{ARCH}$ & $45.1(88.2)$ & $100(100)$ \\
\hline ARMA & $100(100)$ & $95.1(99.0)$ \\
\hline & $\operatorname{Pr}$ (reject independence/non-independent) & $\operatorname{Pr}($ reject determinism/chaotic model $)$ \\
\hline LoGISTIC & $100(100)$ & $1.50(0.85)$ \\
\hline HENON & $100(100)$ & $1.27(0.01)$ \\
\hline LORENZ & $100(100)$ & $1.52(0.25)$ \\
\hline
\end{tabular}

Note: The results for the larger data set are given in parenthesis. H-independence test uses pboots.-values.

To conclude this numerical analysis we consider the behavior of our methodology confronted with data obtained from noisy-chaotic systems which constitute an interesting border case. Hommes and Manzan (2006) have stated the question about whether the null hypothesis of low-dimensional noisy chaos has been clearly rejected within the relevant economic literature. Certainly, this is a crucial query for those researchers interested in economic dynamics. In order to cast some light on this issue we have applied the procedures derived from permutation entropy to the chaotic asset pricing model with heterogeneous beliefs proposed by Brock and Hommes (1998) and buffeted with different levels of dynamic noise ${ }^{14}$. In this way, we investigate if our tests are sensitive to an insignificant amount of noise that contaminates main dynamics. Central to the model, the agents endogenously switch (with high intensity) between different beliefs about future asset prices. The

\footnotetext{
${ }^{14}$ We follow the steps given in Hommes and Manzan (2006) to simulate the noisy model.
} 
stochastic nonlinear dynamic model is

$$
\begin{aligned}
x_{t} & =\frac{1}{R} \sum_{h=1}^{4} n_{h, t}\left(g_{h} x_{t-1}+b_{h}\right)+\sigma \epsilon_{t} \\
n_{h t} & =\frac{e^{\beta U_{h, t-1}}}{\sum_{j=1}^{4} e^{\beta U_{j, t-1}}} \\
U_{h, t-1} & =\left(x_{t-1}-R x_{t-2}\right)\left(g_{h} x_{t-3}+b_{h}-R x_{t-2}\right) .
\end{aligned}
$$

Here the noise term $\epsilon_{t}$ is standard normally distributed with $\sigma$ the standard deviation of the dynamic noise component. In this way, the deterministic skeleton ${ }^{15}$ of the original model is contaminated with dynamic noise. $x_{t}$ stands for the deviation of prices of the risky asset from its benchmark fundamental value; $n_{h, t}$ denotes the discrete choice fraction of agents using belief type $h ; U_{h, t-1}$ represents the profit generated by strategy $h$ in the previous period; $g_{h}$ and $b_{h}$ characterize the linear belief with one time lag of strategy $h$; and finally $R>1$ is the constant gross risk free rate $^{16}$.

Table 5 shows the results of our new test for chaos applied to time series (2000 observations ${ }^{17}$ ) generated by the model (12), (13) and (14) in the deterministic case $(\sigma=0)$ and stochastic cases $(\sigma \neq 0)$ which contain a deterministic skeleton. For illustrative purposes and with the intention of comparing our results with those obtained with Shintani and Linton's Lyapunov exponent based test, Table 5 also collects the results of Shintani and Linton's test for chaos. To complete Table 5 we have also included the results of the 0-1 measure of complexity $(\mathrm{H})$ and the bootstrapped p-values of our test of independence.

As commented in the introduction, Shintani and Linton (2004) stablished a statistical framework for testing the chaotic hypothesis based on the estimated Lyapunov exponents and a consistent variance estimator. This allows them to present a feasible one-sided test for chaos in time series. The statistic is

$$
L E=\frac{\hat{\lambda}_{M}}{\sqrt{\hat{\Phi} / M}}
$$

where $\hat{\lambda}_{M}$ is a neural network estimator of the largest Lyapunov exponent, $M$ is the number of evaluation points used for estimating $\hat{\lambda}_{M}$, and $\hat{\Phi}$ is the consistent estimator of the asymptotic variance of the Lyapunov exponent. Asymptotically, $L E$ is distributed as a standard normal random variable. In order to estimate $L E$, a neural network estimation of nonlinear AR model has

\footnotetext{
${ }^{15}$ See Figure 2 for delay plots of the model.

${ }^{16}$ Readers interested in the model are recommended to consult Brock and Hommes (1998).

${ }^{17}$ In consequence we have set $m=5$.
} 


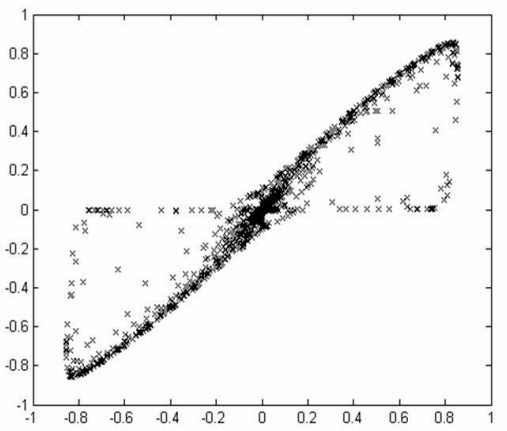

(a) Deterministic : $\sigma=0$

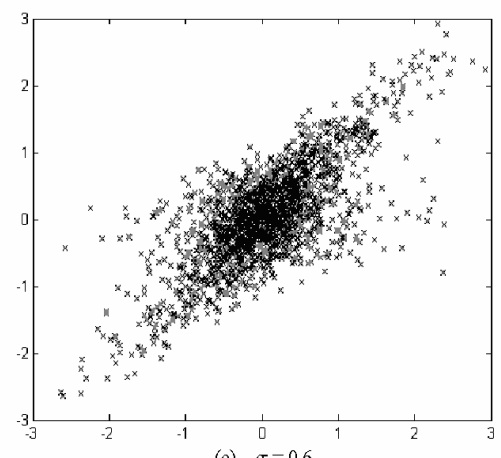

(c) $\sigma=0.6$

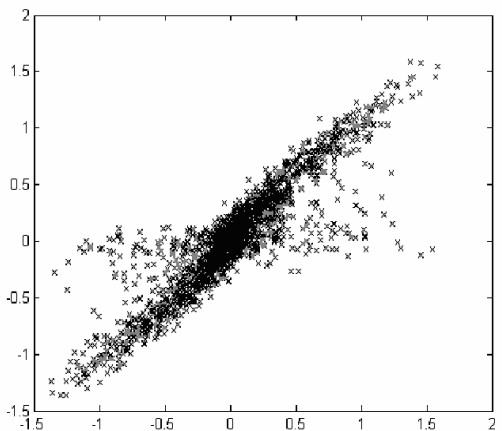

(b) $\sigma=0.3$

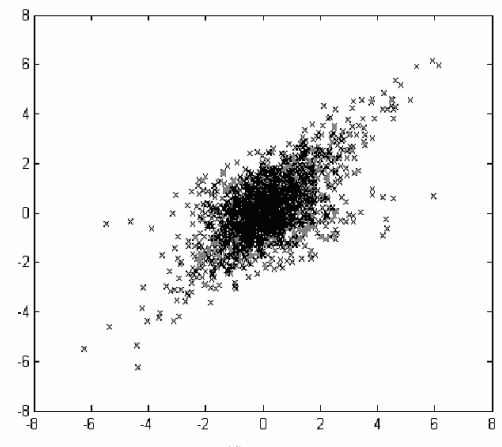

(d) $\sigma=0.9$

Figure 2: Delay plots $\left(x_{t-1}, x_{t}\right)$ of the nonlinear model (12) for the deterministic case and for 3 different noise levels; (a) shows (a projection of) the strange attractor of the deterministic skeleton $\sigma=0$. Parameters are: $R=1.01, \beta=90, g_{1}=b_{1}=0, g_{2}=0.9, b_{2}=0.2, g_{3}=0.9, b_{3}=-0.2, g_{4}=1.01$ and $b_{4}=0$.

to be conducted. In this case we have used three lags in the estimation of the neural network (corresponding to the true dimension 3 of the system) and four hidden units. The nonlinear AR estimation has been conducted under R-statiscal software, and the rest of the statistic has been estimated under GAUSS 6.0.

As an overview of the general dynamics of the simulated processes, we observe that when the deterministic skeleton dominates the dynamic process, the $0-1$ measures are clearly below 1 . For high noise levels $(\sigma=0.8, \sigma=0.9$ and $\sigma=1$ ), our measure is near its limit point since the deterministic skeleton is fully melted with intense noise and hence it is not detected by our 0-1 measure. However our bootstrap test for independence clearly detects dependence structure even with intense noise. On the other hand, the new test for determinism points to a non-rejection of the null for noise's levels below 0.7; that is, deterministic structure is being captured. These results contrast with those obtained with LE test as the null of chaos is now not rejected in presence of noise levels bellow $\sigma=0.2$. Summing up, if the researcher needs to detect noisy deterministic 
systems, our test, even though not constructed for this aim, seems to be a useful tool.

Table 5. $\alpha_{\underline{1}}$, LE tests for chaos and bootstrap independence test in a low-dimensional noisy model

\begin{tabular}{ccccccc} 
& $H$ & pboots. - value & $\hat{\alpha}_{1}$ & \multicolumn{4}{c}{$L E$} \\
\hline$\sigma=0.0$ & 0.41 & $(0.00)$ & 0.05 & $(0.30)$ & 0.135 & $(1.00)$ \\
$\sigma=0.05$ & 0.43 & $(0.00)$ & 0.05 & $(0.25)$ & 0.122 & $(1.00)$ \\
$\sigma=0.1$ & 0.44 & $(0.00)$ & 0.10 & $(0.25)$ & 0.112 & $(1.00)$ \\
$\sigma=0.2$ & 0.55 & $(0.00)$ & 0.12 & $(0.23)$ & 0.102 & $(1.00)$ \\
$\sigma=0.3$ & 0.66 & $(0.00)$ & 0.13 & $(0.11)$ & -0.043 & $(<0.001)$ \\
$\sigma=0.4$ & 0.74 & $(0.00)$ & 0.14 & $(0.15)$ & -0.023 & $(<0.001)$ \\
$\sigma=0.5$ & 0.81 & $(0.00)$ & 0.18 & $(0.11)$ & -0.036 & $(<0.001)$ \\
$\sigma=0.6$ & 0.86 & $(0.00)$ & 0.11 & $(0.19)$ & -0.044 & $(<0.001)$ \\
$\sigma=0.7$ & 0.90 & $(0.00)$ & 0.13 & $(0.12)$ & -0.092 & $(<0.001)$ \\
$\sigma=0.8$ & 0.92 & $(<0.001)$ & 0.20 & $(<0.001)$ & -0.194 & $(<0.001)$ \\
$\sigma=0.9$ & 0.93 & $(<0.001)$ & 0.20 & $(<0.001)$ & -0.172 & $(<0.001)$ \\
$\sigma=1.0$ & 0.95 & $(0.001)$ & 0.20 & $(<0.001)$ & -0.128 & $(<0.001)$ \\
\hline
\end{tabular}

Note: Bootstrap independence test, and the Tests for Determinism $\left(\hat{\alpha}_{1}\right)$ and $(L E)$ for various levels of noise $\sigma$. pboots.-value refers to the bootstraped p-value under the null of independence.

\section{Application to financial data}

Numerous models that are able to generate chaos in economic variables have been developed. In this section we focus our empirical research on those related with finance. As mentioned in the preceding section, Brock and Hommes (1998) provided an asset pricing model with heterogeneous beliefs of agents that produces chaos in stock prices. Shintani and Linton (2004) have investigated the possibility of chaos in the U.S. financial market using stock price series. Particularly, they use daily observations on the Dow Jones Industrial Average (DJIA), $P_{t}$, ranging from January 3, 1928 to October 18, 2000, in order to test for chaos in return series. Returns are defined as the difference of logarithm of the stock price index $\left(R_{t}=\Delta \log P_{t}\right)$.

In parallel, a considerable number of monetary models have shown that they are capable of generating chaotic behavior in exchange rates (see, for example, De Grauwe et al. 1993, Szpiro 1994; and see also De Grauwe and Grimaldi (2006) for a model with heterogeneity of agents that is based on the switching mechanism of Brock and Hommes (1998)). Fernández-Rodríguez et al. 
(2005) have looked for evidence of chaos in three exchange rate's returns: the French franc, the German mark and the Canadian dollar, all against the U.S. dollar. The three analyzed series go from January 4, 1971 to December 31, 1998. Kyrtsou and Serletis (2006) have also tested for deterministic structure in Canadian exchange rate returns extending the sample period till February $14,2000$.

In this section we investigate the possibility of deterministic structure in the four financial time series above mentioned. Because it is well-known that the volatility measures such as the absolute returns, $\left|R_{t}\right|$, have higher autocorrelation compared to the return time series, $R_{t}$, we also apply our tests to the power of absolute returns, $\left|R_{t}\right|^{d}, d=1 / 2,1,1.5$ and 2 .

Results of the 0-1 measure for complexity and of the deterministic test are presented in Table 6 along with their corresponding $p$-values. Because of the number of observations, we have fixed $m=6$. Firstly we note that the $0-1$ measure yields, in all cases, values very close to 1 indicating high complexity. Fortunately, by means of the bootstrap test for independence, we can observe that, despite the proximity to the unit, the null of independence is clearly rejected at all levels for the DJIA and the Canadian Dollar returns. It is straightforward to understand that simple deterministic dynamics are unable to achieve high values of $\mathrm{H}(\mathrm{m})$, rather the contrary. We emphasize, however, that the found dependence is not compatible with a deterministic explanation since our tests for determinism clearly reject the null. As a result, it is possible to search for dependence in their transformations. For the Canadian currency we observe that dependence is found in the transformed data set and so it would be worth for the researcher to model the variance, while the opposite happens for the DJIA data set. For the French Franc and the German Mark the null of independence is accepted (non-rejected) for returns. As can be observed the null of determinism is clearly rejected. Transformed data sets reveal that structure (dependence) is found in their corresponding transformations for the French Franc but not for German Mark. Further research in second moments of the franc is therefore advisable.

As already mentioned, other studies have tested for deterministic components in the same data sets. Our findings on the DJIA data set are against a deterministic explanation of stock returns which strengthens those conclusions given in Shintani and Linton ${ }^{18}$. As regards Canadian exchange rates, our results are incompatible with a deterministic skeleton in returns and in its transformations. The same outcomes were found in Kyrtsou and Serletis (2006), but not in

\footnotetext{
${ }^{18}$ These results agree with them, except for the fact that our rejection is at $1 \%$ level and they cannot reject at this level.
} 
Fernández-Rodríguez et al. (2005). In this later paper deterministic chaotic behavior was also found in the returns of the French franc/US dollar, but not in the German mark exchange rate. In contrast, our outcomes show that, not only the German mark' returns are incompatible with chaos, but also the French franc' returns.

Note that the results of the $0-1$ procedure for power absolute returns are identical because of the above stated permutation entropy's property of invariance with respect to different monotonic transformations of the original time series. Note as well that permutation entropy $H(m)$ is always clearly above the values shown in Table 4, hinting that returns and transformed returns seems to be far from having a low-dimensional noisy structure. 
Table 6. Tests for Determinism and Independence in Financial Returns

\begin{tabular}{|c|c|c|c|c|}
\hline & DJIA & French Franc & German Mark & Canadian Dollar \\
\hline \multicolumn{5}{|c|}{$x_{t}=R_{t}$} \\
\hline $\mathrm{H}$ & $0.991(<0.001)$ & $0.992(0.49)$ & $0.992(0.50)$ & $0.991(<0.001)$ \\
\hline$\hat{\alpha}_{1}$ & $0.223(<0.001)$ & $0.224(<0.001)$ & $0.221(<0.001)$ & $0.229(<0.001)$ \\
\hline \multicolumn{5}{|c|}{$x_{t}=\left|R_{t}\right|^{0.5}$} \\
\hline $\mathrm{H}$ & $0.997(0.65)$ & $0.990(0.03)$ & $0.991(0.34)$ & $0.992(<0.001)$ \\
\hline$\hat{\alpha}_{1}$ & $0.226(<0.001)$ & $0.223(<0.001)$ & $0.230(<0.001)$ & $0.229(<0.001)$ \\
\hline \multicolumn{5}{|c|}{$x_{t}=\left|R_{t}\right|$} \\
\hline $\mathrm{H}$ & $0.997(0.65)$ & $0.990(0.03)$ & $0.991(0.76)$ & $0.992(0.01)$ \\
\hline$\hat{\alpha}_{1}$ & $0.229(<0.001)$ & $0.223(<0.001)$ & $0.224(<0.001)$ & $0.220(<0.001)$ \\
\hline \multicolumn{5}{|c|}{$x_{t}=\left|R_{t}\right|^{1.5}$} \\
\hline $\mathrm{H}$ & $0.997(0.65)$ & $0.990(0.03)$ & $0.991(0.76)$ & $0.992(0.01)$ \\
\hline$\hat{\alpha}_{1}$ & $0.230(<0.001)$ & $0.226(<0.001)$ & $0.225(<0.001)$ & $0.226(<0.001)$ \\
\hline \multicolumn{5}{|c|}{$x_{t}=\left|R_{t}\right|^{2}$} \\
\hline $\mathrm{H}$ & $0.997(0.65)$ & $0.990(0.03)$ & $0.991(0.76)$ & $0.992(0.01)$ \\
\hline$\hat{\alpha}_{1}$ & $0.229(<0.001)$ & $0.227(<0.001)$ & $0.227(<0.001)$ & $0.225(<0.001)$ \\
\hline
\end{tabular}

Note: The p-value of the 0-1 procedure $(\mathrm{H})$ under the null of independence is in parentheses.

Other p-values are considered under the null of determinism.

\section{Conclusions}

This paper has introduced a new way for testing for deterministic dynamicis, including low dimensional chaos and also we have introduced a bootstrapped and consistent test for independence. These statistical procedures critically rely on the concept of entropy which, as presented here, is formulated in terms of symbols. These symbols are obtained, in this case, from the ordinal patterns found in a given data set. We emphasize that we do not work directly with the actual observed values (which are real numbers), rather we take the number of order patterns in the observed series as a potential measure of its complexity. Focusing on symbols, we are able to detect global 
properties of the data generating process such as dependence (independence) and determinism (stochastic nature). We find an intrinsic common characteristic for any deterministic process that does not hold for a stochastic one. Economists have been worried about finding chaos in economic variables. Unfortunately, few tests for determinism against nondeterminism are available. Prior studies, mainly tangent methods and direct methods, test for chaos via estimation of the largest Lyapunov exponent. We take a new different way; if the process is deterministic (chaotic or regular), then our permutation entropy based test is able to detect it. The statistical methods presented in this paper are fast in computing times, simple and powerful. In addition, the test for independence and the test for determinism are nonparametric, invariant under monotonic transformations, and free regarding the discrete or continuous nature of the generating process. As a result the only parameter that has to be freely fixed by the researcher is the embedding dimension $(m)$, which sharply contrasts with other existing procedures that require the practitioner to select several free parameters.

On the other hand, the performance of the tests in moderate sample sizes and for well-known time series are certainly satisfactory. As a valuable application, we have put into operation the permutation entropy tests to study various time series of financial returns which have been recently

analyzed with distinct methodologies. In all cases, we strongly reject the hypothesis of chaos in favor of a nondeterministic structure.

\section{Acknowledgment}

This research has been supported by grant 861-2009-2010 from the Social Sciences and Humanities Research Council of Canada. Mariano Matilla-García was partially supported also by grant MTM2008-03679 from Ministerio de Ciencia e Innovación and FEDER. Manuel Ruiz Marín was partially supported by MEC (Ministerio de Educación y Ciencia), grant MTM2009-07373 and by Fundación Séneca of Región de Murcia

\section{Bibliography}

Bajo-Rubio, O., Fernández-Rodríguez, F. Sosvilla-Rivero, S., 1992. Chaotic behaviour in exchange -rate series. First results for the Peseta_U.S. Dollar case, Economics Letters 39, 207-211.

Bandt, C., Keller, G. Pompe, B., 2002. Entropy of interval maps via permutations. Nonlinearity 
13, 1595-1602.

Barnett, W.A., Gallant, A.R, Hinich, M.J., Jungeils, J.A, Kaplan, D.T., Jensen, M.J., 1997. A single blind controlled competition among tests for nonlinearity and chaos. Journal of Econometrics $82,157-192$.

Binder, P.M., Igarashi, R., Seymour, W., Takeishi, C., 2005. Determinism test for very short time series. Physical Review E 71, 036219, 1- 4.

Brock, W.A., Dechert, W., Scheinkman, J.A., LeBaron, B., 1996. A test for independence based on the Correlation Dimension. Econometric Reviews 15, 197-235

Brock, W.A. Hommes, C.H., 1998. Heterogeneous beliefs and routes to chaos in asimple asset pricing model, Journal of Economic Dynamics and Control 22, 1235-1274.

Brock, W.A., Malliaris, A.G., 1989. Differential Equations, stability and chaos in dynamic economics. Elsevier, Amstemdam.

Brock, W. A., Sayers, C., 1988. Is the Business Cycle Characterized by Deterministic Chaos?. Journal of Monetary Economics 22, 71-90.

Casdagli, M., Eubank, S. Farmer, J.D., Gibson, J., 1991. State space reconstruction in presence of noise. Physica D 51, 52-98.

De Grauwe, P., Dewachter, H., Embrechtes, M., 1993. Exchange rate theories. Chaotic models of the Foreign Exchange Markets. Blackwell: Oxford.

De Grauwe, P., Grimaldi, M., 2006. Exchange rate puzzles: a tale of switching attractors. European Economic Review 50, 1-33.

Eckmann, J.P., Kamphorst, S.O., Ruelle, D., Ciliberto, S., 1986. Lyapunov exponents from time series. Physical Review A 34, 4971-4979.

Eckmann J.P., Ruelle D., 1985. Ergodic theory of chaos and strange attractors. Reviews of Modern Physics 57, 617-650.

Elliot, G., Granger, C.W.J. , Timmermann, A., 2006. Handbook of Economic Forecasting, Vol 1. Elsevier, Amsterdam.

Fernández-Rodríguez F., Sosvilla-Rivero, S. , Andradra-Félix, J., 2005. Testing chaotic dynamics via Lyapunov exponents. Journal of Applied Econometrics 20, 901-930.

Frank M, Stengos T., 1988. Some evidence concerning macroeconomic chaos. Journal of Monetary Economics 22, 423-438.

Gençay R., 1996. A statistical framework for testing chaotic dynamics via Lyapunov exponents. Physica D 89, 261-266. 
Gilmore, C.G., 1993. A new test for chaos. Journal of Economic Behavior and Organization $22,209-237$.

Gottwald, G.A., Melbourne, I., 2004. A new test for chaos in deterministic systems. Proccedings of the Royal Society of London A 460, 603-611.

Gottwald, G.A., Melbourne, I., 2005. Testing for chaos in deterministic systems with noise. Physica D, 100-110.

Granger, C.W.J., 2008. Non-linear Models: Where Do We Go Next - Time Varying Parameter Models? Studies in Nonlinear Dynamics \& Econometrics 12, 1-9.

Granger, C.W.J., Lin J.L., 1994. Using the mutual information coefficient to identify lags in nonlinear models. Journal of Time Series Analysis 15, 371-84.

Granger, C.W.J., Maasoumi, E., Racine, J. 2004. A Dependence Metric for possibly nonlinear processes. Journal of Time Series Analysis 23, 649-669.

Grassberger, P., Procaccia I., 1983. Measuring the strangeness of strange attractors. Physica 9D, 189-208.

Hamilton, J.D., 1994. Times Series Analysis. Princeton University Press, New York.

Hao, B. , Zheng, W., 1998. Applied Symbolic Dynamics and Chaos. World Scientific, Singapore.

Hommes, C., Manzan, S., 2006 Comments on testing for nonlinear structure and chaos in economic time series. Journal of Macroeconomics 28, 169-174.

Hong, Y., White, H., 2005. Asymptotic distribution theory for nonparametric entropy measures of serial dependence. Econometrica 73, 837-901.

Kantz H., 1994. A robust method to estimate the maximal Lyapunov exponents from time series. Physics Letters A 185, 77-87.

Kaplan, D.T., Glass, L., 1992. Direct Test for Determinism in a Time Series. Physical Review Letters 68, 427-430.

Kyrtsou, C., Serletis, A., 2006. Univariate tests for nonlinear structure. Journal of Macroeconomics 28, 154-168.

Maasoumi, E., Racine, J., 2002. Entropy and predictability of stock market returns. Journal of Econometrics 107, 191-312.

Matilla-García, M., 2007. Nonlinear dynamics in energy futures. The Energy Journal 28, 7-30.

Matilla-García, M., Queralt, R., Sanz, P., Vázquez, F.J., 2004. A Generalized BDS Statistic. Computational Economics 24, 277-300.

McCaffrey D.F., Ellner S., Gallant R., Nychka D.W., 1992. Estimating the Lyapunov exponent 
of a chaotic system with nonparametric regression. Journal of the American Statistical Association $87,682-695$.

Nychka, D., Ellner, S., McCaffrey, D., Gallant, A. R., 1992. Finding Chaos in Noisy Systems. Journal of the Royal Statistical Society Series B 54, 399-426.

Robinson, P.M., 1991. Consistent nonparametric entropy-based testing. Review of Economic Studies 58, 437-453.

Rohatgi, V.K., 1976. An Introduction to Probability Theory and Mathematical Statistics. John Wiley \& Sons, New York.

Rosenstein M., Collins J.J., De Luca C., 1993. A practical method for calculating largest Lyapunov exponents from small data sets. Physica D 65, 117-134.

Scheinkman J., LeBaron B., 1989. Nonlinear dynamics and stock returns. Journal of Business $62,311-337$.

Schreiber T., Kantz, H., 1995. Noise in chaotic data: Diagnosis and treatment. Chaos 5, $133-142$.

Shannon, C.A., 1948. A mathematical theory of communication. Bell System Technical Journal $27,379-423,623-656$.

Shintani, M. , Linton, O., 2004. Nonparametric neural network estimation of Lyapunov exponents and a direct test for chaos. Journal of Econometrics 120, 1-33.

Skaug H. J. , Tjøstheim D., 1996. Measures of Distance Between Densities with Application to Testing for Serial Independence. In Robinson P.M., Rosenblatt, M. (Eds.). Time Series Analysis in Memory of E. J. Hannan, Springer-Verlag, New York, 363-377.

Szpiro G.G., 1994. Exchange rate speculation and chaos inducing intervention. Journal of Economic Behavior and Organization 24, 363-368.

Takens F., 1981. Detecting strange attractors in turbulence. In Dynamical Systems and Turbulence, Rand D, Young L. (Eds.). Springer-Verlag, Berlin, 366-381.

Ullah, A., 1996. Entropy, divergence and distance measures with econometric applications. Journal of Statistical Planning and Inference 49, 137-162.

Wolf A., Swift JB., Swinney H., Vastano J., 1985. Determining Lyapunov exponents from a time series. Physica D 16, 285-317.

Yao, Q., Tong H., 1998. A bootstrap detection for operational determinism. Physica D 115, 49-55. 